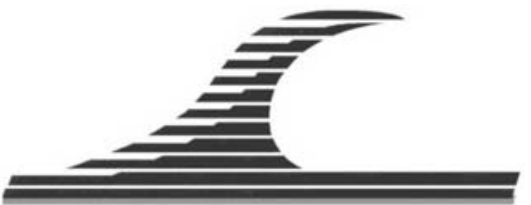

\title{
Caractérisation des sédiments et morphologie de l'embouchure du fleuve Comoé (Grand-Bassam, Côte d'Ivoire)
}

\section{Kouassi Laurent ADOPO ${ }^{1}$, Kouakou Lazare KOUASSI ${ }^{1}$, Ama Valérie Irma WOGNIN ${ }^{2}$, Sylvain MONDE ${ }^{2}$, Kouamé AKA ${ }^{2}$}

${ }^{1}$ Laboratoire de Géologie Marine, Sédimentologie et Environnement, Centre de Recherche en Ecologie, Université d’Abobo-Adjamé, 01 BP 10588 Abidjan 01, Côte d'Ivoire. l_adopo@yahoo.fr ; k_lazare@yahoo.fr

${ }^{2}$ Laboratoire de Géologie Marine et Sédimentologie ; UFR-Sciences de la Terre et des Ressources Minières, Université de Cocody,

22 BP 582 Abidjan 22, Côte d'Ivoire.

ama_valerie@yahoo.fr ; smonde@hotmail.fr ; akaraphael@yahoo.fr

\section{Résumé :}

Les analyses granulométriques et les levés bathymétriques effectués à l'embouchure du fleuve Comoé, entre 2005 et 2007, ont permis de caractériser les sédiments et la morphologie du fond de cette embouchure, sujette à d'importantes modifications. Sur la plage, les sédiments sont constitués de sables majoritairement grossiers à moyens alors que dans l'estuaire on rencontre essentiellement des vases et des sables vaseux. Par ailleurs, on enregistre une plus grande variété de minéraux sur la plage que dans l'estuaire.

Les épaisseurs annuelles de dépôts au niveau de l'estuaire sont faibles devant les dépôts marins. Cette étude a permis de réaliser les premières cartes bathymétriques de l'estuaire du fleuve Comoé.

Soumis le 16 juin 2008, accepté le 29 décembre 2008, en ligne le 29 décembre 2008. La seule version examinée est celle écrite en français. La ou les autres versions n'étant pas examinées par le comité de rédaction de la revue, sont donc publiées sous l'entière responsabilité du ou des auteurs.

A TRANSLATED VERSION IN ENGLISH IS AVAILABLE ONLINE

Pour citer cet article :

ADOPO K.L., KOUASSI K.L., WOGNIN A.V.I., MONDE S., AKA K. (2008). Caractérisation des sédiments et morphologie de l'embouchure du fleuve Comoé (Grand-Bassam, Côte d’Ivoire). Revue Paralia, $\mathrm{n}^{\circ}$ 1, pp 2.1-2.10. 


\section{Introduction}

Les estuaires, zones de rencontre entre le fleuve et la mer, présentent une dynamique particulaire assez complexe (LE NORMANT, 1995 ; GOUBERT et al., 2008 ; DI MATTEO \& MILLI, 2008). En effet, les dépôts des particules transportées par les eaux fluviales et marines façonnent la morpho-structure des paysages estuariens.

En Côte d’Ivoire, les embouchures des grands fleuves (Comoé, Bandama, Sassandra et Cavally) connaissent un dynamisme de plus en plus accentué qui se traduit généralement par des déplacements des points de confluence fleuve-mer (ABE, 1995 ; WOGNIN et al., 2007). Au niveau de l'embouchure du fleuve Comoé à Grand-Bassam, zone de rencontre entre l'Océan Atlantique, la lagune Ebrié et le fleuve Comoé, la dynamique hydrosédimentaire est encore plus spectaculaire si bien que les dépôts successifs de sédiments donnent naissance à un banc de sables qui rompt le contact naturel entre le fleuve et l'océan (KOFFI \& ABE, 1991). Ceci a pour conséquence le comblement accéléré de l’embouchure, la modification significative des paramètres physico-chimiques et le développement rapide des plantes aquatiques sur la lagune (SANKARE \& ETIEN, 1991 ; SANKARE et al., 1991). Des travaux de dragage ont été effectués pour ouvrir cette embouchure qui s'est toujours refermée quelques années plus tard (TASTET, 1979 ; ABE et al., 1996). Ainsi, des opérations d'ouverture ont respectivement eu lieu en 1987, 1990 et 2005 après les colmatages observés en 1975, 1989 et 2003. La fermeture de cette embouchure entraîne le confinement du milieu estuarien. ABE (1995) indique que la fermeture de l'embouchure du fleuve Comoé aurait des conséquences à la fois sédimentologique (envasement), chimique (confinement, dessalure), écologique et économique (pêche, tourisme) considérables.

Malgré les dangers inhérents à la fermeture de l'embouchure du fleuve Comoé avec le temps, peu d'études tentent d'expliquer les processus hydrodynamiques et sédimentologiques qui s’y déroulent (ABE et al., 1996 ; ADOPO et al. 2008).

La connaissance de la dynamique sédimentaire dans les estuaires permet d'appréhender les processus d'envasement et de colmatage dont ces milieux sont le siège. Elle contribue aussi à la compréhension des caractères physicochimiques et biologiques de ces environnements complexes qui représentent des zones de transit, de transfert et de stockage (AVOINE et al., 1986; SANCHEZ \& LEVACHER, 2007 ; AMEY et al., 2007).

La présente étude a pour objectif de caractériser la nature des sédiments et la morphologie de l'embouchure du fleuve Comoé. 


\section{Caractérisation des sédiments et morphologie de l'embouchure}

du fleuve Comoé (Grand-Bassam, Côte d'Ivoire) : 2.3

\section{Site et méthodes}

\subsection{Localisation, cadre géologique et climatique}

Le secteur estuarien du fleuve Comoé est à l'extrémité orientale de la lagune Ebrié. Il est situé entre $5^{\circ} 12^{\prime}$ et $5^{\circ} 14^{\prime}$ de latitude Nord et $3^{\circ} 42^{\prime}$ et $3^{\circ} 44^{\prime}$ de longitude Ouest. Cette zone constitue le plus vaste estuaire du littoral ivoirien (KOFFI \& ABE, 1991). La zone d'étude couvre les environs de l'île Morin au Nord, le pont de Moossou à la confluence du fleuve Comoé et de la lagune Ebrié, l'île Bouet et le cordon littoral au Sud. Elle inclue le bras du fleuve Comoé jusqu'au village de Yaou au Nord-Est (figure 1).

La zone estuarienne est alimentée par le fleuve Comoé qui draine tout l'arrière pays et couvre une superficie de $78000 \mathrm{~km}^{2}$.

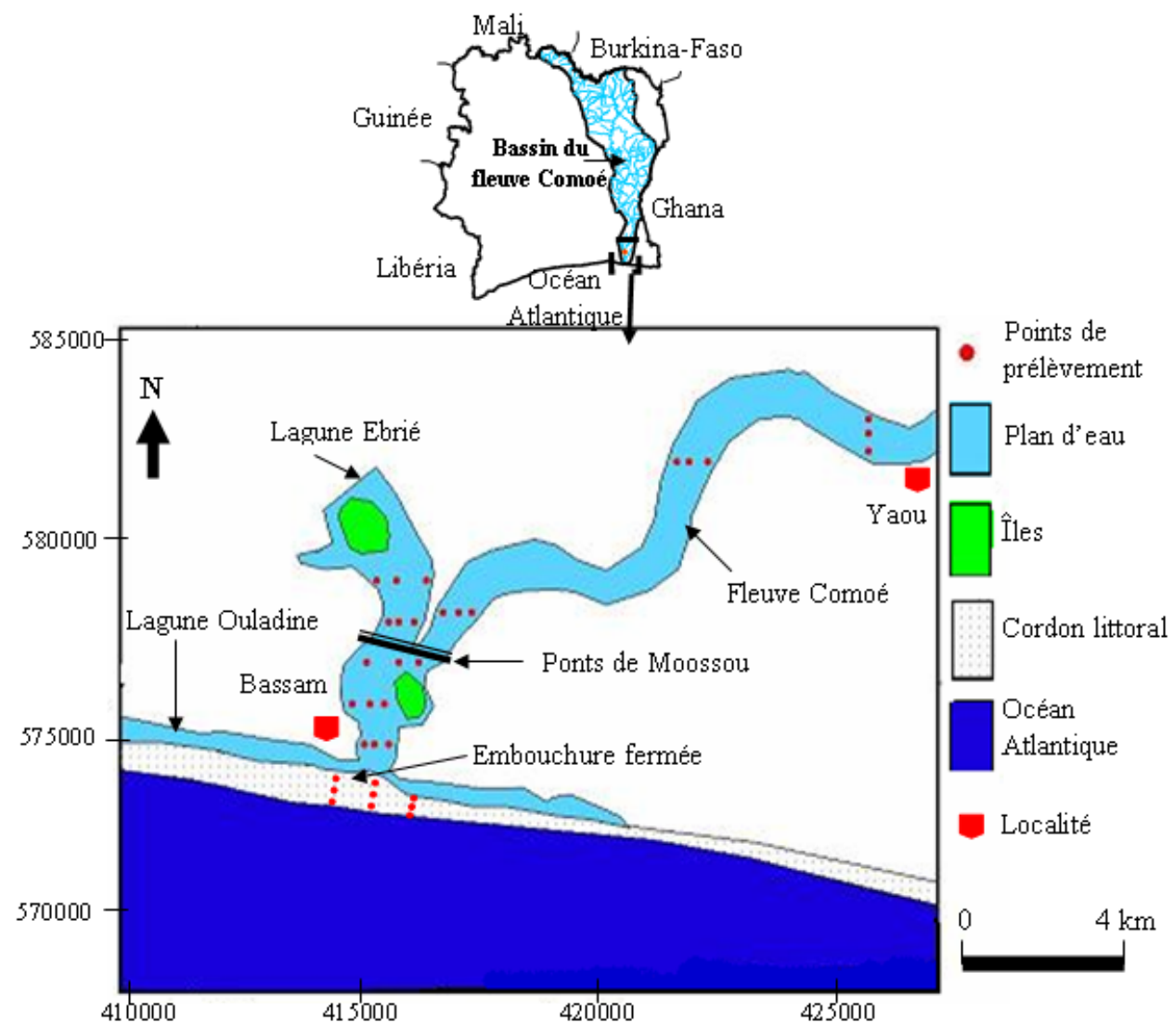

Figure 1. Localisation de l'estuaire du fleuve Comoé à Grand-Bassam.

\subsection{Méthodes d'étude}

Pour déterminer les caractéristiques granulométriques des sables à l'embouchure du fleuve Comoé, 24 échantillons de sédiments ont été prélevés sur huit radiales dans l'estuaire (voir figure 1) à l'aide d'une benne Van Veen. Neuf échantillons 
de sable ont également été prélevés à l'embouchure, sur trois profils de plage au niveau du haut, moyen et bas estran. La morphologie de la plage est relativement monotone aux voisinages de l'embouchure. Les sédiments ont été analysés selon une technique décrite par SAAIDI (1991). Les matières organiques et les débris coquilliers ont été éliminés respectivement à l'aide de l'eau oxygénée 30\% et de l'acide chlorhydrique 50\%. Après élimination de la fraction inférieure à $63 \mu \mathrm{m}$, chaque échantillon de sable a subi une analyse granulométrique à sec sur une colonne de 16 tamis (série AFNOR). Les sables de l'estuaire ont été caractérisés à travers la moyenne granulométrique (Mz), le skewness (Sk) et l'indice de classement (So), déterminés à l'aide des méthodes de FOLK (1974).

L'étude de la morphologie du fond de l'estuaire a été effectuée par l'intermédiaire de levés bathymétriques, réalisés à l'aide d'un échosondeur de type Lowrance, modèle LMS-160. Quatre levés topographiques ont été réalisés sur un cycle saisonnier aux voisinages de l'embouchure pour déterminer les quantités de sables engraissés ou érodés sur la plage. Les cartes sédimentologiques et les cartes de positionnement ont été établies à l'aide des logiciels ArcView 3.3 et SURFER 8.2.

\section{Résultats}

\subsection{Description des faciès sédimentaires}

L'analyse granulométrique des sédiments superficiels de l'estuaire du fleuve Comoé met en évidence trois faciès lithologiques. Il s’agit des sables, des vases et des sédiments "mixtes". Les vases sont de couleur variable et se localisent aux voisinages des berges. Les vases sont noirâtres et très riches en matières organiques. La crème de vase présente une couleur grisâtre et est riche en matières organiques. Les sédiments "mixtes" sont constitués de sables vaseux et de vases sableuses. Leur coloration varie du noir olive au gris olive. Ils contiennent à la fois des débris végétaux et des débris coquilliers.

La granulométrie des sables contenus dans les sédiments prélevés révèle que les grains sont fins à grossiers. Dans l'ensemble, la moyenne granulométrique des sables est comprise entre 67 et $765 \mu \mathrm{m}$ (tableau 1). Les indices de classement se situent entre 0,58 et 1,12. Ceci indique que les sables dans l'estuaire sont modérément classés à mal classés. Les indices d’asymétrie (skewness) s’étendent de 0,06 à 0,25 indiquant une forte asymétrie vers les petites tailles.

Au niveau de la plage, la granulométrie des sables augmente, de façon générale, du haut estran (525 $\mu \mathrm{m}$ en moyenne) au bas estran (918 $\mu \mathrm{m}$ en moyenne). Les indices d'asymétrie sont négatifs $(-0,13)$ au haut estran et positifs $(0,12)$ au moyen et au bas estran. Les indices de classement sont de l'ordre de 0,29. 
Tableau 1. Granulométrie des sables à l'embouchure du fleuve Comoé.

\begin{tabular}{llllll} 
& & \multicolumn{4}{c}{ Moyenne granulométrique $(\boldsymbol{\mu m})$} \\
\cline { 3 - 6 } & & Minimum & Maximum & Moyenne & Ecart type \\
\hline \multirow{2}{*}{ Estuaire } & 67 & 765 & 329 & 231,12 \\
\hline \multirow{3}{*}{ Plage } & Haut estran & 469 & 708 & 525 & 86,34 \\
& Moyen estran & 532 & 824 & 756 & 74,57 \\
& Bas estran & 863 & 1012 & 918 & 62,60 \\
\hline
\end{tabular}

\subsection{Minéralogie des sables}

Le spectre minéralogique des sables de l'embouchure du fleuve Comoé est caractérisé par un ensemble de minéraux lourds et de minéraux légers.

Au niveau de l'estuaire, les principaux minéraux lourds rencontrés dans la fraction sableuse sont essentiellement du grenat (2\%), de l'amphibole (2\%), du pyroxène (4\%), de la tourmaline jaune (3\%) et de l'épidote (2\%). Parmi les minéraux légers, on retrouve le quartz (77\%), le mica (7\%) et le feldspath (3\%).

Le cortège de minéraux lourds observés sur la plage est composé de l'amphibole (1\%), l'épidote (3\%), le leucoxène (3\%), la sidérite (1\%), la sillimanite $(2 \%)$, le rutile (4\%), le chrysobéryl (2\%), l’ilménite (14\%), la limonite (4\%), la monazite (3\%), la tourmaline (3\%), le pyroxène (2\%), le diopside $(3 \%)$, l’or $(1 \%)$, le grenat (3\%) et le zircon (3\%). Le quartz est le minéral léger le plus rencontré (48\%) dans les échantillons.

\subsection{Morphologie du fond de l'estuaire du fleuve Comoé}

L’examen détaillé de la bathymétrie de l'estuaire du fleuve Comoé fait apparaître une morphologie de fond relativement complexe (figure 2).

L'échelle des couleurs et les valeurs des isobathes mettent en évidence le chenal principal du fleuve qui a une direction NE-SO. Les profondeurs diminuent de l'amont vers l'embouchure du fleuve. Cependant, les profondeurs les plus élevées (14 m) s'observent au niveau des deux ponts de Moossou. Le bassin de l'estuaire présente, dans l'ensemble, des pentes relativement faibles qui varient entre 0,4 à 2,6\%. Aux voisinages de l'embouchure, l'organisation générale de la morphologie des fonds montre des zones peu profondes entaillées par des dépressions.

La superposition des cartes bathymétriques réalisées respectivement en 2005 et en 2007 ont permis d'apprécier les évolutions de fonds de l'estuaire. Dans l'ensemble, l'estuaire subit un phénomène de dépôt dont l'épaisseur moyenne est d'environ $20 \mathrm{~cm}$ sur les deux années (2005-2007) soit $10 \mathrm{~cm}$ par an. 


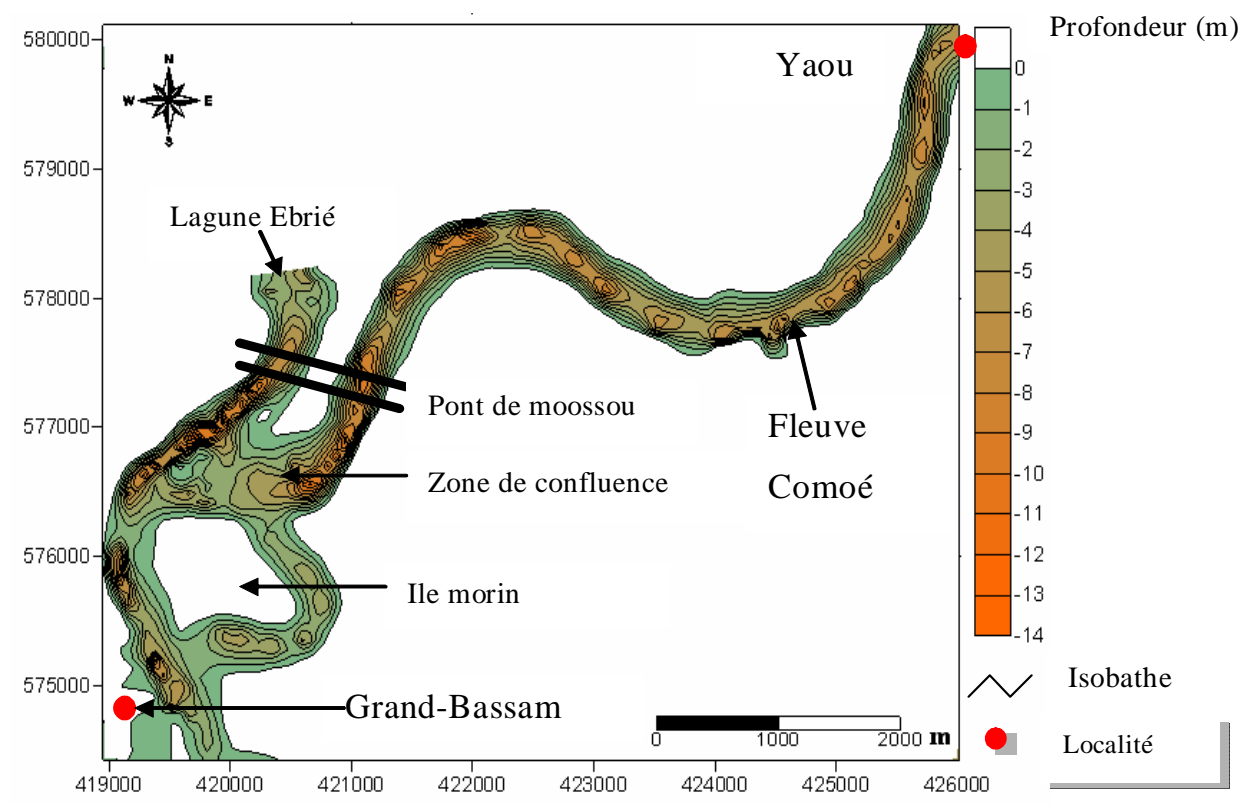

Figure 2. Bathymétrie de l'estuaire du fleuve Comoé (août 2005).

\subsection{Evolution des profils de plage}

Le suivi des profils de plage, sur un cycle saisonnier, a permis d'apprécier les mouvements sédimentaires au droit de l'embouchure du fleuve Comoé. Afin de permettre les comparaisons, les profils relevés sur l'axe de l'embouchure gardent sensiblement la même longueur au cours des saisons (figure 3). La morphologie de la plage subit des fluctuations plus importantes au bas estran. La comparaison des profils de juin 2006 à mars 2007 révèle une érosion au haut estran et un engraissement au moyen et au bas estran. Les épaisseurs de dépôts atteignent $1 \mathrm{~m}$ par endroits. L’épaisseur moyenne annuelle de dépôts est de l'ordre de 60 cm.

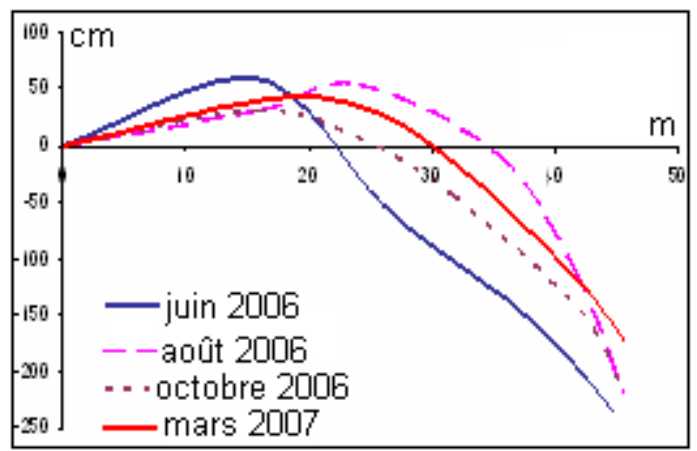

a : Evolution saisonnière

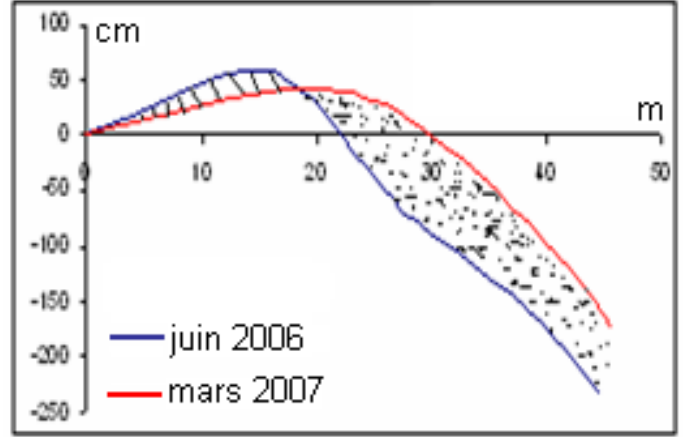

b : Evolution entre juin 2006 et mars 2007

Figure 3. Evolution du profil de plage à l'embouchure du fleuve Comoé entre juin 2006 et mars 2007. 


\section{Discussion}

Les sédiments du secteur estuarien de l'embouchure du fleuve Comoé, où se rencontrent la lagune Ebrié et le fleuve Comoé, renferme majoritairement des vases et des sables vaseux. En revanche, sur la plage, on rencontre essentiellement des sables grossiers à moyens.

L’analyse minéralogique des sables révèle la présence de minéraux lourds et de minéraux légers aussi bien sur la plage que dans l'estuaire. Toutefois, on observe une diversité spécifique des minéraux plus importante au niveau de la plage. Cela peut s'expliquer par la dérive littorale qui déplace différents types de sables de l'Ouest vers l'Est de la Côte d'Ivoire (AKA, 1991). Les minéraux rencontrés dans l'estuaire font partie de ceux décrits par BROCHE et al. (1977) dans la partie supérieure du bassin versant du fleuve Comoé.

Les épaisseurs de dépôts (10 cm par an en moyenne) enregistrées au cours de cette étude au niveau de l'estuaire sont faibles pour occasionner la fermeture rapide de l'embouchure. Ces faibles épaisseurs de dépôts sont en accord avec le transport solide en suspension du fleuve Comoé qui est de l'ordre de 72000 tonnes par an selon ADOPO et al. (2008).

En revanche, la dynamique sédimentaire marine est assez significative et occasionne des dépôts dont l'épaisseur peut atteindre $1 \mathrm{~m}$ par an au droit de l'embouchure du fleuve Comoé. Cette quantité de dépôt constituerait l'une des principales causes de la fermeture rapide de l'embouchure du fleuve Comoé. ABE et al. (1996) rapportent que le caractère irrégulier des débits du fleuve Comoé pourrait expliquer, en partie, cette fermeture. En effet, selon ces auteurs, la réduction significative des débits de ce cours d'eau pendant les périodes d'étiage, notamment au cours de ces dernières décennies du fait de l'influence des fluctuations climatiques, limite la pénétration des eaux continentales dans l’océan. Il se crée alors une barrière qui favorise les dépôts de sédiments.

\section{Conclusion}

Les analyses granulométrique et minéralogique ont montré que les sédiments rencontrés à l'embouchure du fleuve Comoé sont constitués de sables grossiers à fins au niveau de la plage tandis que le secteur estuarien renferme une importante proportion de vases et de sables vaseux. Les épaisseurs annuelles de dépôts au niveau de l'estuaire sont faibles devant les dépôts marins. Les actions hydrodynamiques constituent, dans ce contexte, un facteur déterminant dans la fermeture de l'embouchure du fleuve Comoé.

Les travaux se poursuivent dans le but d'établir un modèle de la sédimentation de l'estuaire du fleuve Comoé. 


\section{Remerciements}

Cette recherche a fait l'objet d'un soutien financier de la Fondation Internationale pour la Science (IFS, Suède). Nous adressons nos sincères remerciements à cette institution pour sa contribution à nos travaux.

\section{Références bibliographiques}

$\mathrm{ABE}$ J. (1995). Etude comparative de la dynamique sédimentaire aux embouchures des fleuves du littoral ivoirien. Proc. Int. Conf. "Costal change 95" Bordomer-IOC, Bordeaux, pp 347-363.

ABE J., BAKAYORO S., BAMBA S, CISSOKO S. (1996). L'hydrologie de l'estuaire du Comoé à Grand-Bassam (Côte d'Ivoire). Agronomie Africaine. Journal Ivoirien d'Océanologie et Limnologie. Vol. 8, n 3, pp 201-212.

ADOPO K.L., KOUASSI K.L., WOGNIN A.V.-I. (2008). Spatial Distribution and Characterisation of the Sediments of the Estuary of Comoe River (GrandBassam, Côte D’ivoire). European Journal of Scientific Research, Vol. 20, $\mathrm{n}^{\circ} 4$, pp 817-827.

AKA K. (1991). La sédimentation quaternaire sur la marge de la Côte d'Ivoire: Essai de modélisation. Thèse de Doctorat d'Etat ès Sciences Naturelles, Univ. d'Abidjan, Côte d'Ivoire, $\mathrm{n}^{\circ}$ 146, 320 p.

AMEY K.B., BEDJA K., NEGLO K. (2007). Distribution longitudinale de la granulométrie du sable littorale togolais : grain moyen, sorting index et skewness. Journal des Sciences pour l'Ingénieur, $\mathrm{n}^{\circ}$ 8, pp 1-8.

AVOINE J., DUBRULLE L., LARSONNEUR C. (1986). La dynamique sédimentaire dans les estuaires de la baie de seine - conséquences sur l'environnement. La Baie de Seine (GRECO-MANCHE) - Université de Caen, IFREMER, Actes de Colloques n 4, pp 183-192.

BROCHE J., CASSANOVA R., LOUP G. (1977). Atlas des minéraux en grain, identification par photographies en couleurs. Société pour le développement minier de la Côte-d'Ivoire, 173 p.

DI MATTEO A., MILLI A. (2008). Morphological, bathymetric and sedimentological surveys used to assess the coastline defensive measures. Int. J. Environ. Sci. Tech., Vol. 5, n 3, pp 415-424

FOLK R.L. (1974). Petrology of sedimentary rocks. Hemphills, Austin, Texas, $182 \mathrm{p}$. 
GOUBERT E., FRENOD E., PEETERS P., THUILLIER P., VESTED H.-J., BERNARD N., VERON V., ALLANO L. (2008). Caractérisation $d u$ fonctionnement hydrosédimentaire de l'estuaire de la Vilaine à partir d'études bathymétriques et altimétriques (Altus). $\mathrm{X}^{\mathrm{èmes}}$ Journées Nationales Génie Côtier Génie Civil, Sophia Antipolis, pp 81-90. Article disponible en ligne [URL http://www.paralia.fr/jngcgc/10_08_goubert.pdf ]

KOFFI K, ABE J. (1991). Contribution à l'étude des modifications hydrosédimentaires consécutives à la réouverture artificielle de l'embouchure du Comoé à Grand-Bassam. Journal Ivoirien d'Océanologie et Limnologie. Vol. 1, $\mathrm{n}^{\circ} 2$, pp 47-60.

LE NORMANT C. (1995). Modélisation numérique tridimensionnelle des processus de transport des sédiments cohésifs en environnement estuarien. Thèse de doctorat, Institut National Polytechnique de Toulouse ; spécialité physique et chimie de l'environnement. $\mathrm{n}^{\circ} 1002,253 \mathrm{p}$.

SAAIDI E. (1991). Traité de sédimentologie. Pétrographie, environnements sédimentaires. Editions Ellipses, 393 p.

SANCHEZ M., LEVACHER D. (2007). The influence of particle size of the dispersed mineral fraction on the settlement of marine and estuarine muds. GeoMarine Letters, Vol. 27, n 5, pp 303-313.

SANKARE Y., ETIEN N. (1991). Analyse des effets de l'ouverture du chenal de Grand Bassam (estuaire du fleuve Comoé, Lagune Ebrié) sur la macrofaune benthique lagunaire. Journal Ivoirien d'Océanologie et Limnologie, Vol. 1, n 2, pp 81-90.

SANKARE Y., KOTHIAS A.J.-B., KONAN A.A. (1991). Les effets de la réouverture de l'embouchure du fleuve Comoé sur la végétation littorale lagunaire (Lagune Ebrié - Côte d'Ivoire). Journal Ivoirien d'Océanologie et Limnologie, Vol. 1, n² 2, pp 71-79.

TASTET J.P. (1979). Environnements sédimentaires et structuraux quaternaires du littoral du Golfe de Guinée (Côte d'Ivoire, Togo et Bénin). Thèse de doctorat, Université de Bordeaux I, n 621, 175 p.

WOGNIN A.V., MONDE S., AFFIAN K., COULIBALY A., AKA K. (2007). Modèle de circulation des eaux dans l'estuaire du fleuve Bandama en Côte d'Ivoire. Sud Sciences \& Technologies, n 15, pp 5-12. 
2.10 : Revue Paralia - $n^{\circ} 1$ (2008) 